\title{
MEASUREMENTS OF SUBMICRON FOG PARTICLES GENERATED IN A NOZZLE
}

\author{
P. M. SHERMAN \\ Department of Aerospace Engineering, University of Michigan, Ann Arbor, Michigan 48104, U.S.A.
}

(Received 4 June 1974)

\begin{abstract}
Measurements were made of the size and relative number of particles condensed in a "large" supersonic nozzle. Stagnation temperature was held at $528^{\circ} \mathrm{R}$ and stagnation pressure was varied from $-1 / 3$ to $-2 / 3$ atm with the water as the condensing vapor. The mass fraction of water vapor was varied from $\sim 8 \times 10^{-3}$ to $-30 \times 10^{-3}$. The size of particles was determined by measurement of light scattered at two angles, making use of the Mie solution for particles above the maximum size for Rayleigh scattering. The size of particles measured was found to be an order of magnitude larger than previously, with radii from $\sim 200$ to $\sim 700 \AA$. It was found that the initial partial pressure of vapor was the best correlating parameter with particle size over the total pressure range, and that the relative number of particles decreased by an order of magnitude as the mass fraction increased. Static pressure measured in the test section of the nozzle showed an increase with initial mass fraction of vapor which was approximately linear.
\end{abstract}

\section{INTRODUCTION}

When a superheated vapor is expanded rapidly, as in a nozzle or a free jet, it will reach a saturation point as the temperature drops. If the medium is relatively "clean" there will not be sufficient surface for the condensation necessary to maintain equilibrium and the medium will become supersaturated. Further expansion results in condensation from spontaneous (homogeneous) nucleation. In the nozzle the fluid expands by an adiabatic process not unlike that which occurs when free air masses move from a high pressure region to one of lower pressure. The nozzle contour determines the rate of the expansion and hence the rate of condensation. Such condensation is an important consideration in turbines, molecular beams, wind tunnels and combustion.

The classical theory for predicting homogeneous condensation, of Frenkel (1956), which has been employed most extensively for comparison with the experimental measurements cannot predict parameters for any given physical event but has been used for determining qualitative effects of parameters by Griffen and Sherman (1965), Chmielewski and Sherman (1970), and for extending the usefulness of the experimental data. Much more data are needed before any theory can be properly tested. The primary purpose of the present measurements was to obtain new data, in particular, to determine of size of particles condensed in a "large" supersonic nozzle where the process is relatively slow.

There have been several measurements of the size of condensed particles. A direct method of electron microscopy was used for metallic particles by McBride and Sherman (1972). An indirect method was used by Thomann (1966) to infer the size of ice particles in a supersonic wind tunnel by measuring the vapor pressure in the gas sucked into pitot tubes of different diameters and calculating particle trajectories around the pitot tubes as a function of particle size. Rayleigh scattering has been used by Stein and Wegener (1967) and by Clumpner (1970), for measurements at the exit of small nozzles; the light scattering 
method used here has been employed for condensation in air by Durbin (1951) in a hypersonic tunnel.

The theory of light scattering by small particles predicts the angular distribution of intensity and degree of polarization of light scattered by a fog. The general solution was derived by Mie in 1908 and has since been employed in the form of published tables for different materials (different indices of refraction) wave lengths and particle size. The solution can be employed to determine particle size in several different ways. Measurement is easiest and most accurate when relative rather than absolute intensities of light are used. For very small particles $(r<0.02 \lambda)$ light is scattered according to Rayleigh's solution which yields forward and backward scattering in a symmetrical pattern. As the radius of the particles increases, the scattering becomes asymmetrical and a comparison of forward and backward scattering yields the size of the particle from the classical Mie solution. For a particle circumference of the same order of magnitude as the wave length of the light scattered, a unique solution for particle size can be obtained from the ratio of intensities of light scattered at two angles which are symmetrical about the line of incidence. The solution yields particle size as a unique function of the ratio of intensities measured at the two angles, and is independent of the intensity of the light scattered. The solutions are based on the assumptions of single scattering and "clean" flow. Both assumptions have been shown to be good for the supersonic nozzle flow considered. The measurement does, however. represent an average particle size when the particle size is not uniform. Both theory and experiment have indicated (Durbin, 1951; Thomann, 1966; Stein and Wegener, 1967; McBride and Sherman, 1972) that a relatively narrow size distribution can be expected for particles condensed in supersonic nozzles. In one case, Clumpner (1970) estimated a S.D. of $\sim 8 \AA$.

\section{EXPERIMENTAL MEASUREMENTS}

All measurements were made in the continuous supersonic tunnel of the University of California, Berkeley with a nominal Mach 2.7 nozzle (of rectangular cross-section) with a test section region $\sim 5.5$ in. wide $\times 4.6$ in. high. The test station was 25.75 in. downstream of the throat. The stagnation temperature was controlled and kept at $68^{\circ} \mathrm{F}$ and the stagnation pressure was controlled and varied from about $1 / 3$ to $2 / 3 \mathrm{~atm}$. A traverse mechanism in the test section supported both a pitot tube and a screen for focusing. Pressure measurements were made with manometers, and a sensitive dewpointer (Cambridge Systems No. 880) was used to measure the partial pressure of water in the stagnation chamber through a continuous bleed from the settling chamber. Water was bled into the dry air through a hypodermic needle.

The primary measurements made were of the intensity of light scattered in two directions. Figure 1 is a schematic of the optical system employed. Light from a mercury arc lamp (GE BH6 air cooled) with an adjustable slit was focused in the center of the test section at an angle of $\sim 70^{\circ}$ to the direction of flow. Two photomultipliers (Hamamatsu TV Co. R212 and RCA 1P21) each in a fixed enclosed housing were attached to an optical bench (oriented $90^{\circ}$ to the direction of flow) which included $4070 \AA$ filters and polaroid screens to permit only light polarized in the plane of the incident beam to be seen by the phototubes. A regulated power supply (Hewlett-Packard-Harrison $6515 \mathrm{~A}$ d.c. set at 750 $\mathrm{V})$ and potentiometer for matching the output of the two tubes were used with electrometers (Keithly No. 600A) for readout. The outputs of the photomultipliers were 


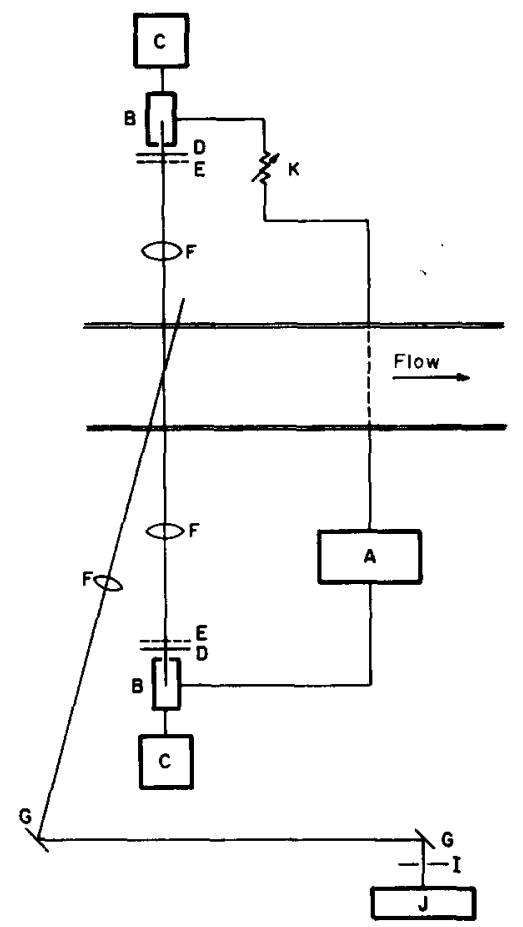

Fig. 1. Schematic of arrangement. A-regulated power supply; B-photomultipliers; C--electrometers; D-filters; E-polaroids; F-lenses; G-front surface mirrors; I-adjustable slit; J-high pressure mercury arc.

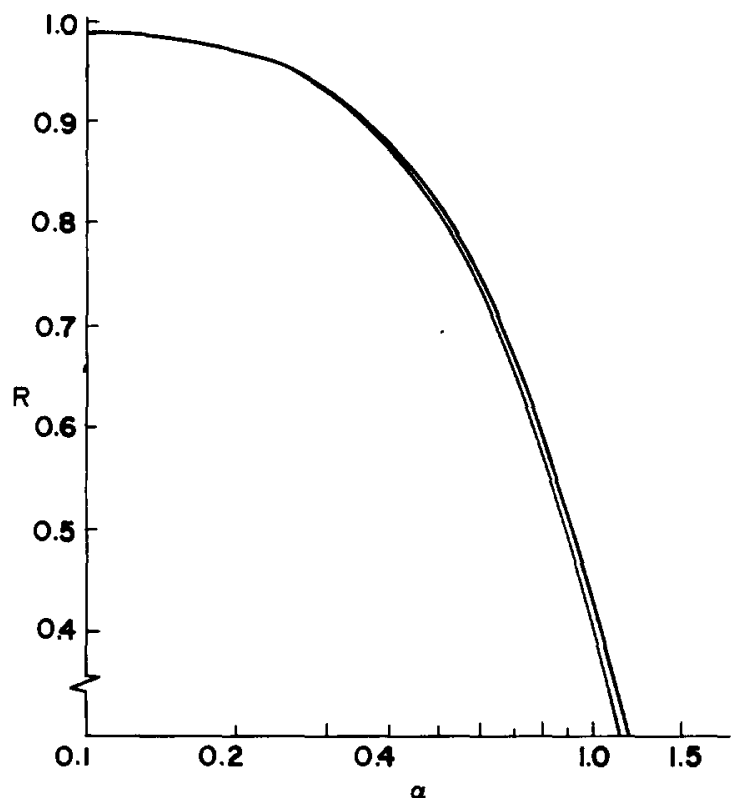

Fig. 2. Ratio of intensity of scattered light, $R$, at $20^{\circ}$ to that at $160^{\circ}$ to the angle of incidence for an index of refraction of 1.2 and 1.33 as a function of non-dimensional particle size, $\alpha$, according to Mie's solution. ( $\alpha$ is the ratio of the circumference of the particle to the wavelength of incident light.) 
recorded and particle size was determined from the ratio of the two signals scattered at 20 and $160^{\circ}$ to the incident beam. Figure 2 shows the relationship of the ratio of the scattered signals, $R$, to the non-dimensional particle radius $\alpha$, where $\alpha$ is the ratio of particle circumference to wave length of incident light. Figure 2 was plotted from tabulated data, Durbin (1951) and NBS (1949), based on the classical solution of Mie.

Although it would be difficult to determine the absolute intensity of light scattered, it is much easier and increases accuracy to employ the ratio of two intensities ( $R$ was measured within -3 per cent). The plot of $R$ vs $\alpha$ is not sensitive to change in either angle or index of refraction for the conditions considered here. As one can see from the curve (Fig. 2) a large error can occur for small $\alpha$ but as $\alpha$ increases the error in $x$ as a result in an error in $R$ decreases very rapidly. The dewpointer was accurate to $\pm 0.5^{\circ} \mathrm{F}$ or -2 per cent in stagnation vapor pressure. Local static pressure was accurate to $\sim 0.001 \mathrm{in}$. $\mathrm{Hg}$; stagnation and pitot pressure to $0.1 \mathrm{in}$. $\mathrm{Hg}$.

A determination of the relative number of particles condensed was also made. For a given particle size, the light intensity scattered is proportional to the number of particles. Therefore if at a reference state of given particle size, intensity from measurements and from the classical Mie solution are noted and compared to the intensity from measurements and the Mie solution at any different chosen particle size, then the change in number of particles relative to the reference state can be determined from the ratio of the normalized measured intensity to the normalized Mie solution intensity. This determination was repeated for a range of values for $\alpha$ from 0.4 to $1 \cdot 1$. A comparison was also made with relative number density computed by assuming all the water vapor was condensed by the time it reached the test section, an assumption frequently employed for a small mass fraction of vapor, Stein and Wegener (1967), Clumpner (1970).

\section{RESULTS}

Measurements made of the average size of the particles condensed in a "large" supersonic nozzle indicate that larger particles than previously measured are indeed obtained. The slower expansion in a larger nozzle permits slower nucleation and extended growth. Previous measurements Thomann (1966), Stein and Wegener (1967) of size of particles obtained when water vapor condenses in a supersonic air stream have been done in small nozzles (with expansion times approximately a factor of 50 times faster than in the present nozzle) and the size determined were an order of magnitude smaller.

Figure 3 shows the measured particle size as a function of mass fraction of vapor, $k_{0}$, in terms of the non-dimensional parameter, $\alpha$, the ratio of the circumference of the particle to the wave length of light employed, for several stagnation pressures. For the region of small $\alpha$, the change in $r$ with $k_{0}$ is small compared with that for larger $\alpha$. It is interesting that if the measurements are plotted in terms of the initial partial pressure of the vapor as in Fig. 4 (instead of the usual mass fraction as in Fig. 3), the curves for different stagnation pressure approach each other. The initial partial pressure appears to make the most sense as a single correlating parameter. Figure 4 shows that the particle size increases with the initial partial pressure of the vapor; and for low initial partial pressure of vapor $(<0.5$ in. $\mathrm{Hg}$ ) as the amount of carrier gas increases, the size of particle increases. (Curve A is for the highest initial total pressure or the largest mass fraction of carrier gas at a given partial pressure.) A region seems to be reached however $(\sim 450 \AA)$ when an increase in mass of carrier gas (initial total pressure of mixture) makes little difference in particle size. Previous work, Thomann (1966), (for a very small nozzle and mass fraction of 


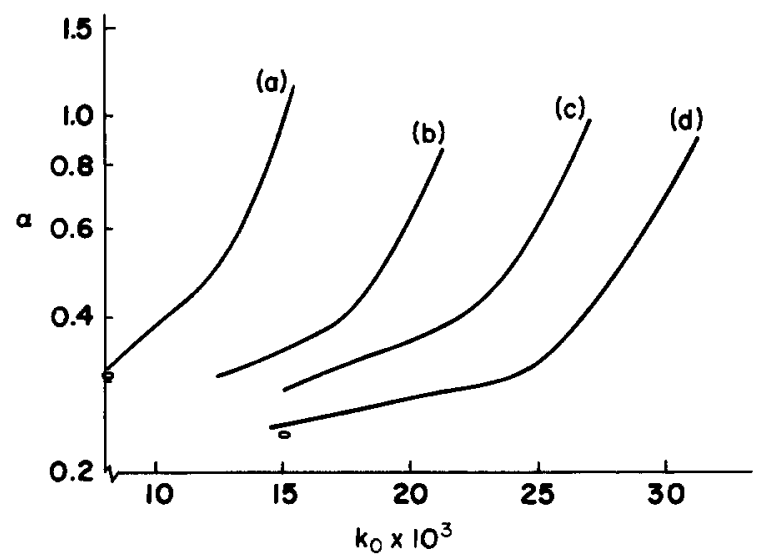

Fig. 3. Change in non-dimensional particle size as a function of mass fraction, $k_{0}$, of water vapor in air. Stagnation temperature was $528^{\circ} R$. Stagnation pressure was $22 \cdot 8,15 \cdot 7,12 \cdot 7,11 \cdot 1 \mathrm{in}$. $\mathrm{Hg}$. for curves A, B, C. D, respectively.

vapor) indicated no change in particle radius with mass fraction. It appears from Fig. 3 that the present measurements are not inconsistent with this since, for conditions where small particles arise, the change in particle radius with mass fraction becomes relatively small.

The classical theory of homogeneous nucleation developed by Frenkel (1956) among others is based on the liquid drop model which assumes that bulk concepts (such as bulk latent heat and surface tension) can be extended down to clusters composed of a relatively small number of molecules. The theory can give reasonable results but they of course are strongly dependent on the physical constants used. In particular, the calculated results are radically dependent on surface tension (which appears as a cube in an exponential) and the way in which surface tension changes with the size of very small particles is not clear. The effect of variations in physical properties has been previously demonstrated, Griffin and Sherman (1965). Small adjustments in the physical properties provide for possible matching of almost any data. However the trends predicted are of interest. A previously

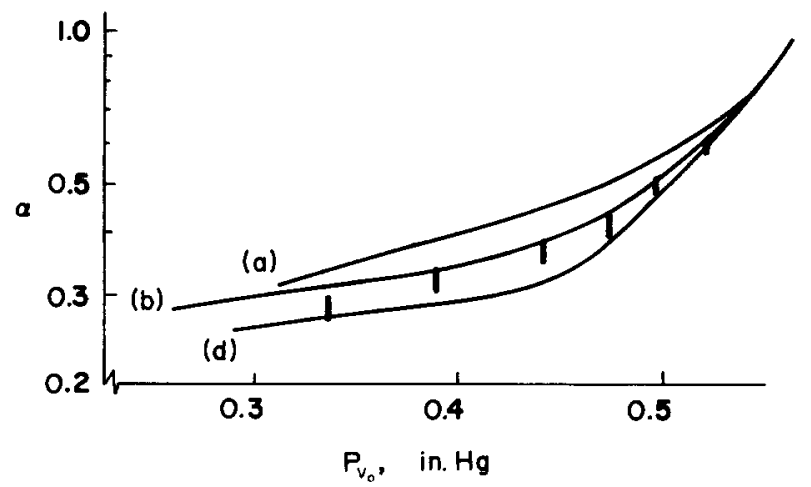

Fig. 4. Particle size as a function of initial vapor pressure $p_{v 0}$. Curves A, B, D, are for the same conditions as in Fig. 3. Curve C of Fig. 3 is indicated by the vertical lines showing approximate size of experimental error. 
employed program, Chmielewski and Sherman (1970), based on the classical liquid drop theory for calculating homogeneous condensation was used with the assumption of complete condensation at the test station, to approximate particle size for several conditions. The points calculated appear circled in Fig. 3. (One for conditions of curve A and one for curve D. More calculations were not completed because of economic consideration.)

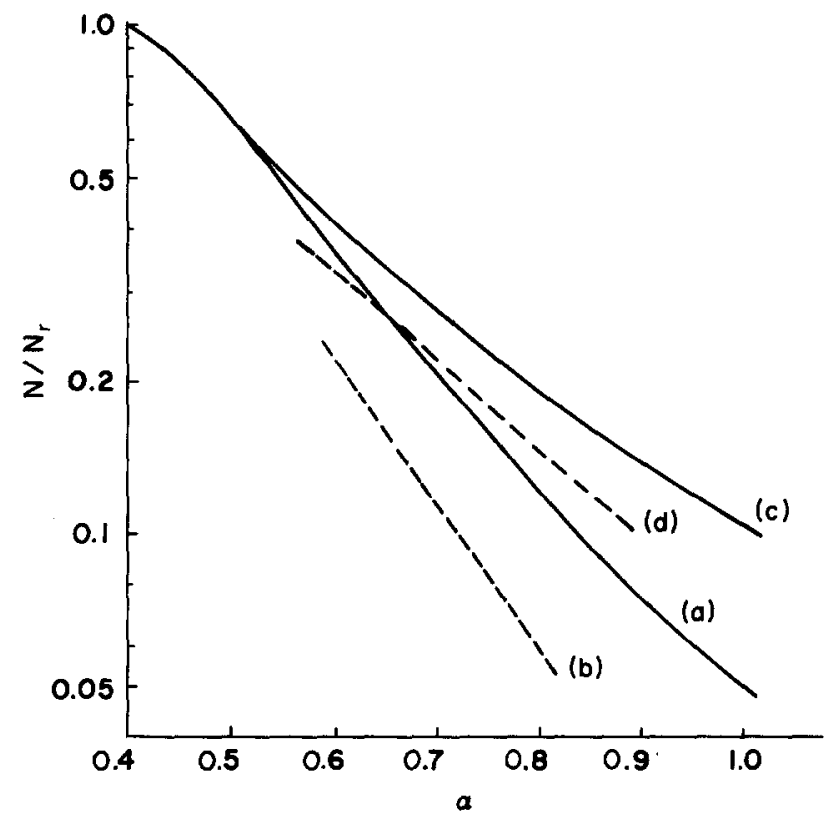

Fig. 5. Relative number of particles as a function of particle size. Curves $A$ and $B$ are from measurements. Curves $C$ and $D$ are computed assuming all vapor is condensed.

While the size of the particles were determined from the ratio of light scattered at two angles, a relative measure of the number density of particles was determined from the measurements of intensity of light scattered at one angle. The theoretically computed intensity of light scattered at a given angle is a function of $\alpha$ for a given number of particles - or per particle. As $\alpha$ increases, the computed intensity of the light scattered increases. The intensity of scattered light measured, however, depends on both $\alpha$ and the number of particles, $N$, per unit volume, which also may change. The intensity at an arbitrary reference $\alpha$ was considered as a reference and the fractional change in $N$ was determined by normalizing the measured intensity at a given $\alpha$ by the intensity computed from the Mie theory for the same $\alpha$ and dividing by the intensity measured at the reference $\alpha$ normalized by the intensity from the Mie theory for the reference $\alpha$. Curves A and B of Fig. 5 show the relative change in number of particles $N / N_{\text {ref }}$ with $\alpha=0.4$ as the arbitrary reference state. Curve A corresponds to conditions for Curve A in Fig. 3 and Curve B corresponds to conditions for Curve D in Fig. 3. Curves corresponding to other stagnation pressures (Curves B and C of Fig. 3) fall between A and B.

The relative number of particles can also be estimated if one assumes that all of the vapor condenses in the nozzle before reaching the test section. The number density of particles at the test section can be written as $N=k_{0} \rho / \rho_{L}\left(\pi r^{3} 4 / 3\right)$ where $\rho$ is the total density 
of the gas mixture, $k_{0}$ is the initial mass fraction vapor, $\rho_{L}$ is the density of the condensed drop and $r$ is the radius of the drop, or using some reference state one could compare the number of particles to that of the reference state by

$$
\frac{N}{N_{\text {ref }}}=\frac{k_{0}}{k_{0_{\text {ref }}}} \frac{r_{\text {ref }}^{3}}{r^{3}} \frac{\rho}{\rho_{\text {ref }}} \frac{\rho_{L_{\text {ref }}}}{\rho_{L}}
$$

If it is further assumed, based on previous work, McBride and Sherman (1971), that $T /$ $T_{f} \sim p / p_{f} ;$ (where $T$ and $p$ are test section temperature and pressure respectively and $T_{f}$ and $p_{f}$ are test section temperature and pressure for the same flow but with no condensation) then for the range of $0<k_{0}<0.03$, the ratio of the test section density, $\rho$, to test section density with no phase change, $\rho_{f}$, will depart from 1 by a maximum of 3 per cent. If $\rho$ and $\rho_{L}$ are then considered constant, $N / N_{\text {ref }}$ is $-\left(k_{0} r_{\text {ref }}{ }^{3}\right) /\left(k_{0_{\text {ret }}} r^{3}\right)$ where $k_{0}$ and $r$ are determined from measured values. Curves $C$ and $D$ in Fig. 5 show this value of $N / N_{\text {rit }}$ as a function of $\alpha$ with the same conditions as Curves $\mathrm{A}$ and $\mathrm{B}$ respectively.

In general, the number of particles condensed decreases with an increase in initial mass fraction of vapor (for given initial conditions). This suggests that the higher partial pressure

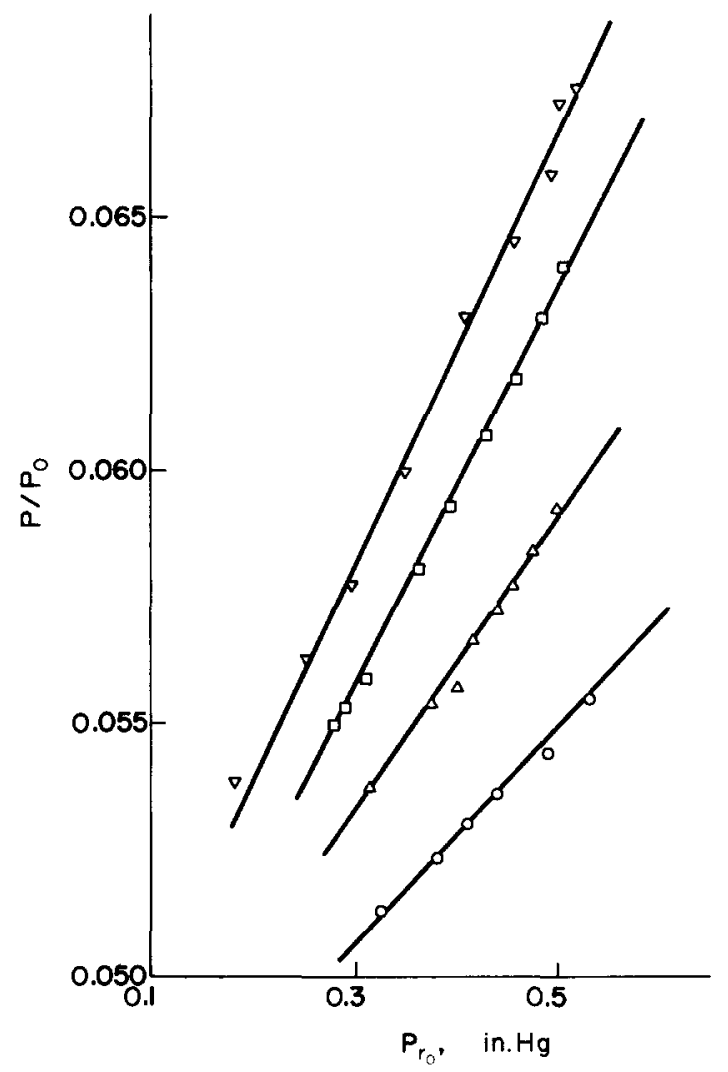

Fig. 6. Test section pressure divided by stagnation chamber pressure as a function of initial partial pressure of water vapor, with stagnation chamber pressure as a parameter. 
of the vapor (for the higher vapor mass fraction) makes for faster growth and hence faster cut-off of nucleation which yields fewer particles growing to a larger size.

Figure 6 shows the measured test section pressure (divided by stagnation chamber pressure) as a function of initial partial pressure of vapor with the initial total stagnation pressure as a parameter. The different slopes are indications of vapor pressure becoming increasing fractions of total pressure with decreasing stagnation chamber pressure. The stagnation chamber pressures used were $22 \cdot 8,15 \cdot 7,12 \cdot 7$ and $11 \cdot 1$ in. $\mathrm{Hg}$. (The zero initial partial pressure of vapor intercept yielded a Mach number of 2.68.)

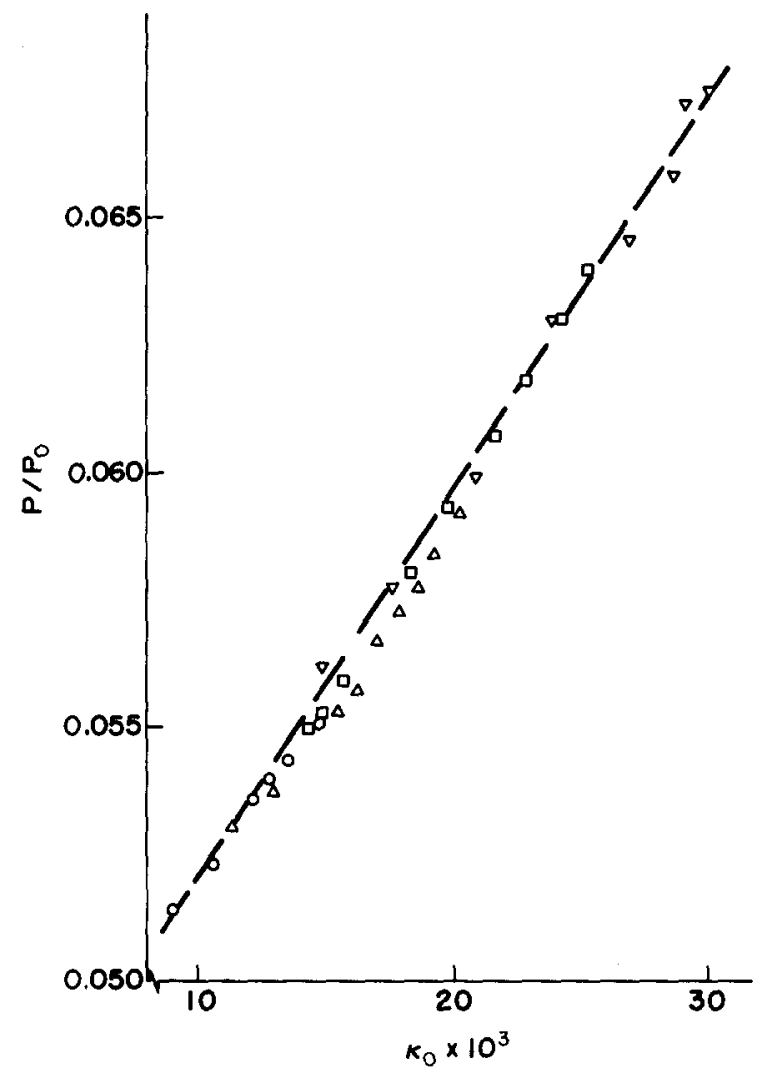

Fig. 7. Test section pressure divided by stagnation chamber pressure as a function of initial mass fraction of vapor. Dashed line is computed approximation from equation (3).

Figure 7 shows how the measured test section pressure (divided by stagnation chamber pressure) changes with the initial mass fraction of the vapor. The pressure at the test station is shown to increase approximately linearly with the increase in mass fraction. The increase in pressure appears reasonable since the major effect of the condensation on the flow through the nozzle is the heat added to the carrier gas as a result of the condensation.

It is not obvious why the test station pressure should appear to be close to a linear function of the initial mass fraction of the vapor. However, if one assumes for the region of $k_{0}$ small (for flow "far" downstream) that $p / p_{f} \sim T / T_{f}$, then $\rho / \rho_{f} \sim$ constant and velocity 
$u \sim u_{f}$. Then for one-dimensional flow, $c_{p} T-c_{p} T_{f} \sim k_{0} L$ (where $L$ is latent heat) and

$$
\frac{p}{p_{0}} \sim\left[\frac{k_{0} L}{c_{p} T_{f}}+1\right] \frac{p_{f}}{p_{0}}
$$

or pressure is a linear function of initial mass fraction of vapor. Equation (2) does not match the measurements very well but if one assumes something closer to that previously calculated by McBride and Sherman (1971), namely $p / p_{f} \sim\left(T / T_{f}\right) 0 \cdot 87$, then the curve calculated from the resultant equation

$$
\frac{p}{p_{0}} \sim\left[\frac{L}{c_{p} T_{0}} \frac{T_{0}}{T_{f}} k_{0}+1\right]^{0.87} \frac{p_{f}}{p_{0}}
$$

is very close to the measurements as shown in Fig. 7.

In conclusion several statements can be made.

1. Present measurements show that condensed particles will grow to a relatively large size in a large nozzle which yields a slow expansion. The particles measured here are an order of magnitude larger than in previous measurements.

2. Initial partial pressure of vapor appears to be a good correlating factor for particle size for a range of total pressures.

3. Within the range of these measurements the static pressure increases approximately linearly with the initial mass fraction of vapor. The resultant static pressure can be approximated from the frozen (no phase change) expansion.

4. In general, the number of particles condensed decreases or the growth rate increases as the initial partial pressure of the vapor increases.

Acknowledgements - The author wishes to thank Professor L. Talbot, Professor F. Robben, and the Department of Mechanical Engineering of the University of California at Berkeley for their assistance and gracious and generous provision of the facilities employed.

\section{REFERENCES}

Chilmielewski, T. and Sherman, P. M. (1970) AIAA J. 8 (4), 789.

Clumpner, J. A., (1970) Ph.D. Thesis, Yale University.

Durbin, E. J. (1951) Optical methods involving light scattering for measuring size and concentration of condensation particles in supercooled hypersonic flow. NACA TN 2441

Frenkel, J. (1956) Kinetic Theory of Liquids, pp. 366-400. Clarendon Press, Oxford. (Also published by Dover, New York.)

Griffin, J. L. and Sherman, P. M. (1965) AIAA J. 4 (10), 1813-1819.

McBride, D. D. and Sherman, P. M. (1971) Astronautica Acta 16 (2), 97.

McBride, D. D. and Sherman, P. M. (1972) $A I A A J .10$ (8), 1058.

National Bureau of Standards (January 1949), Tables of Scattering Functions by Spherical Particles, Appl. Math., Ser. 4.

Stein, G. D. and Wegener, P. P. (1967) J. Chem. Phys. 46, 3685.

Thomann, H. (1966) Physics of Fluids. Vol. 9, No. 5, p. 896. (Am. Inst. Physics). 